\title{
Autonomous Model of Software Architecture for Smart Grids
}

\author{
Zenon Chaczko $^{1}$, Shahrzad Aslanzadeh ${ }^{1}$, Alqarni Lulwah ${ }^{1}$ \\ ${ }^{1}$ University of Technology, Sydney, Faculty of Engineering and IT, Sydney, Australia \\ Zenon.Chaczko@uts.edu.au, Shahrzad.Aslanzadeh@uts.edu.au, \\ Luluqarniegmail.com
}

\begin{abstract}
Smart grids are being deployed at global level to ensure energy efficiency. As a result, scalable smart software platforms are required which can be used to incorporate and integrate information coming from various consumers using smart meters. Smart grids are supported by smart software architectures which are supported by cloud platforms. Cloud and Internet-of-Things (IoT) platforms provide scalable resources which can be used to design software infrastructures which allow always-on applications. The report paper explores smart grid and energy efficiency, how cloud and IoT platforms are used to enhance smart software architecture for smart grids, and privacy and security issues that result from the use of clouds.
\end{abstract}

Keywords: Smart Grids; Smart Software Architecture for Smart Grids; Clouds and Clouds Platforms; Smart Software Architectures, and Software Infrastructures

\section{Introduction}

One of the major challenges of the 21 st century is increased energy demand. As such, there is need to ensure energy efficiency which can be realised through the use of renewable energy and smart grid. However, the ability to run a supply-on-demand system that achieves maximum reliability has emerged as a major challenge. In addition, the inability to balance between largely uncontrolled demand and highly controlled supply has emerged as a major setback on power systems. However, the use of operational smart grid has the likelihood of mitigating some of the challenges faced in the energy sector. This is because it can allow greater penetration of variable energy resources realised through a flexible management system. The concept of smart grid has emerged as more reliable compared to traditional grid. Renewable energies can be integrated through smart software grids to achieve energy efficiency. One of the major cases that can elaborate the need for smart grid and renewable energy is the electrical blackout that took place in North America in 2003 affecting more than a hundred power plants and paralysing different activities. Through the use of smart grids, it becomes easy to meet demand needs. In addition, smart grids ensure efficiency 
driven response and reliability. This report paper explores smart software architecture for smart grids with respect to achieving energy efficiency.

In this paper, smart grid and energy efficiency have been explored. In addition, the writer explores application of clouds platforms to enhance smart software architecture for smart grids with respect to energy efficiency. Lastly, issues of privacy and security in smart software architecture for smart grids supported by clouds have been analysed.

\section{Significance of Smart Grid and Energy Efficiency}

Smart grid is the combination of traditional grid with modern technology, control and information technologies (Potter, Archambault \& Westrick 2008). In other words, smart grid incorporates communication technologies and advanced sensors with the objective of ensuring effective use of assets, increased improved reliability, and energy efficiency as well as allowing consumers to have access to dimensional energy services. Yang (2012) adds that smart grid is the process of using computer-based remote control to automate power systems. Through smart grid technology, power can be generated through renewable energy resources (wind and solar) with the aim of meeting power demand without resource waste thus achieving energy efficiency. Potter, Archambault and Westrick (2008), observe that "the use of smarter grid operations allows for greater penetration of variable energy sources through the more flexible management of the system" (p.1). The implication made is that the use of smarter grids allows flexible management systems which can be achieved through demand side management which is a form of temporary storage technology.

Basically, the existing defining features of smart grids indicate that smart grid offer an interface between traditional assets incorporated in a power system and consumer appliances. As a result, a two-way communication is achieved which provides the consumer with many options and effective control of energy usage. Through the use of sensor networks, power systems become responsive to power stimulus such as power failure. Consequently, efficiency in operations is realised especially when handling interruptions across the power systems. Since smart grid incorporates traditional grid with communication technologies, power production is decentralised. For instance, Porter et al. (2008) suggest that advanced metering technologies coupled with improved communication enhance the use of two way metering and sensors which allow decentralised power production. This allows the optimisation of power flows across the existing power system transmission. Consequently, it improves systems reliability and capital expenditures thus realising energy efficiency.

Lu Liang, Li, Lin and Shen (2011) argue that the electrical blackout that was experienced in 2003 across North America could have been countered through the use of smart grids. This is because, compared to tradition grid, smart grids allow effective real-time diagnosis and load balance through automated outage management, selfactivating, and self healing. In addition, energy efficiency is achieved through cost 
effective power generation, and effective transmission, as well as effective distribution. Some components such as smart meters are vital as they not only allow real-time information collection but they relay the collected information related to grid operations and transmission in real-time (Lu Liang, Li, Lin, \& Shen 2011). Moreover, smart devices have the capability to give consumers increased control and awareness thus reducing energy costs and usage (Drake, Najewicz, Watts, \& General Electric Company 2010). Effective deployment of smart grid technologies allow the integration of variable renewable power, reduction of carbon dioxide emission and management of electricity demand.

\section{Software Architecture for Smart Grids}

For smart software architecture to be in a position to support smart grid application, one of the major intrinsic components required is a cloud platform (Simmhan, Cao, Prasanna \& Giakkoupis 2011). Basically, as earlier stated, smart power grids are incorporated with sensors and smart meters which enable two-ways communication. Therefore, software systems that operate demand response optimisation need a platform that can support generation of data and carryout computation. For example, in Los Angeles city, Department of Water and Power is required to continuously analyse streaming information and data from the city consumers. Therefore, Clouds are necessary as they have "advantages of scalable and elastic resources to build a software infrastructure to support such dynamic, always-on applications" (Simmhan, Giakkoupis, Cao \& Prasanna 2011, p. 1). The implication made is that clouds offer a platform that can be used to develop smart software that can support smart grids hence energy efficiency. All data and information streaming from energy smart grids can be stored in clouds as it streams in large scales. In addition, they allow smart grids operations as clouds allow scalability.

To achieve energy efficiency in a smart grid, smart software architecture is designed. This is because the essence of a smart grid is to enhance effective communications and carry out effective transmission and distribution of power (Michaels \& Donnelly 2010). However, smart grid is based on evolving infrastructures which require smart software architecture from time to time. Therefore, there is need to consider software architecture for smart grid to achieve efficiency. Balaraman (2012) suggests that energy efficient smart grid devices have to be built on smart software architecture. As a result, it would be possible to meet the high demand of energy needs at a lower cost. Given that there has been more renewable energy in most states such as UK and Germany, and energy can effectively be managed through upgrading of the existing emerging grids by replacing them with modern smart software architecture which allows efficiency. As a result, it would be possible to offset peak load demand and manage congestion in smart, effective and efficient way.

The increased demand for energy coupled by rapid growing use of new renewable energy has brought changes to power networks landscapes resulting in new challenges. However, smart grids tend to address these issues especially when smart software architectures are used to integrate software that enhance self-management 
on smart grids (Perez, Diaz \& Gonzalez 2012). It is through these architectures supported by cloud platforms (Simmhan, Giakkoupis, Cao \& Prasanna 2011) that renewable energy resources and traditional power grids are integrated with new technological elements and cloud computing infrastructures which allow scalability and autonomous operations along the power system networks. To achieve these, smart software architecture need to be built based on software architectural models which incorporate power network domain. The diagram below as provided by Perez, Diaz and Gonzalez (2012) is Smart Grid Architectural Model which combines autonomous model and smart grid architectural model to form an Autonomous Smart Grid Architectural Model which enhance energy efficiency.

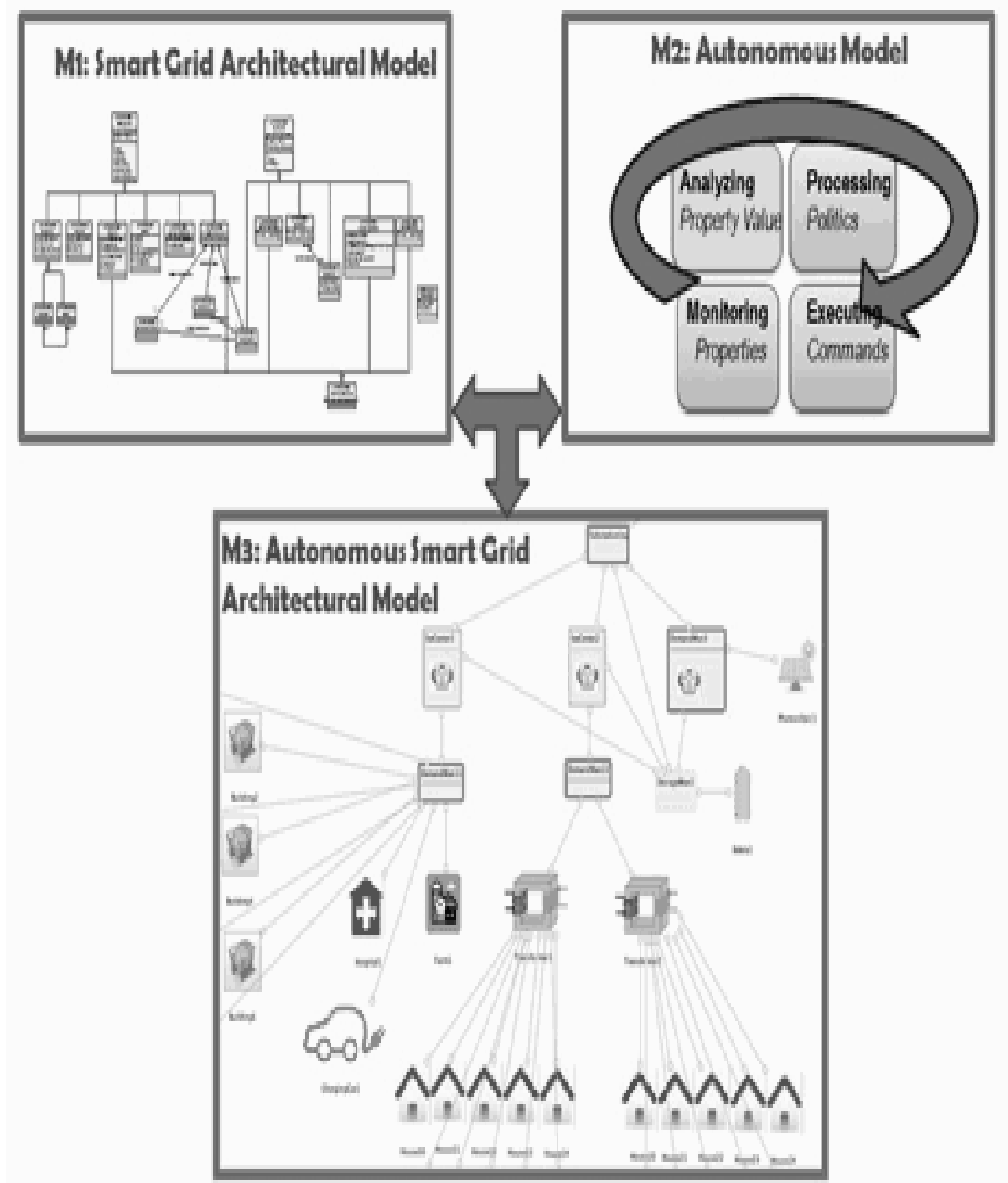


Fig. 1. Proposed Autonomous Smart Grid Architectural Model

Smart Grid software architecture present in clouds allows different stakeholders such as third party service providers, utilities, and consumers to interact. For example, smart meters installed in residential places are based on home area network (HAN) or the building area network (BAN) which gather data related to power usage and relay signals (Simmhan, Giakkoupis, Cao \& Prasanna 2011a). In addition, information on power pricing and energy usage needs to be shared in real-time with power consumers via online portals. This can only be achieved through the use of cloud platforms. Collected data and information which is integrated from different sources need to be accessible and available to third party applications assuming that privacy concerns are met. This benefits smart software developers in developing intelligent applications which meet customer needs. Therefore cloud, either private or public, provides a platform from where data can be shared (Simmhan, Giakkoupis, Cao \& Prasanna 2011). Moreover, clouds provide a platform from where third party applications can be put into place.

One of the major software applications in smart grid architecture is the advanced meter infrastructure (AMI). AMI is defined as a software application that ensures that energy use is measured and the reads communicated over to the utility. In the modern world, companies are upgrading their traditional power grids to smart grids through the use of AMIs which are installed to allow two-way communications between consumers and the utilities in real-time (Simmhan, Cao, Prasanna, \& Giakkoupis 2011). Smart meters increase the volume of data and information available to power systems and utilities. For instance, City of Los Angeles Department of Water and Power has been on the verge of achieving demand-response (DR) optimisation which can only be realised through smart meters built on smart software architecture and supported by cloud platform. Basically, smart enabled grids have the capability to discover faults in power systems. As a result, an average of 20 to $30 \%$ of power is saved compared to traditional grids (Michaels \& Donnelly 2010). AMI offer operating advantages such as outage management, reduced meter reading costs, and granular visibility. To achieve highest peak demand savings, HAN technologies are adopted. Based on a research conducted in California in 2003, households can save $34.5 \%$ through the use of enabling technology.

\section{Security and Privacy Issues in Software Intensive Architectures for Smart Grids}

One of the major challenges being faced in the incorporation of renewable energies into smart grid architecture is cost. This is because multiple technologies are used to ensure that integration which may be costly. Another challenge is security. As noted by Birman, Ganesh, and van Renesse (2012), security is important in ensuring a successful secured smart grid communications. To prevent issues related to insecurity, there is need to ensure report authentication and data integrity. Legacy is experienced 
when there a movement of utilities to smart grids which are based on smart grid software architecture. According to (Simmhan, Giakkoupis, Cao and Prasanna (2011a), not all utilities have the capacity to restructure all the power system. Therefore, coexistence of legacy is allowed. When this happens, privacy and security frameworks are expected to be compatible with existing and new applications. In other others, new applications should be integrated within existing applications without fear for privacy and security issues. However, vulnerabilities and threats that may emerge from insecure legacy smart devices need to be established. Therefore, smart grid applications need to be based on smart software architecture that allows room for privacy and security consideration during the development process. Other methods that can be applied include the use of Smart Grid applications which allow access through Web services, online websites, mobile applications, and local executables (Simmhan et al, 2011a). The applications could also be shared in form of virtual machine image thus ensuring energy usage information security.

A major concern has been raised with the ability of clouds to protect private data and information. As noted by Birman, Ganesh, and van Renesse (2012), current cloud platforms lack protective mechanisms on consumers' private information which has raised consumers concerns. Simmhan et al. (2011a) note that customers can hack communication channels or smart meter thus changing power usage report. In addition, third party software and utilities can access personal information and incorporate power usage data with the aim of forecasting future power demand and load curtailment response. As a result, privacy concerns as a result of security issues need to be addressed while designing smart software architecture for smart grids supported by cloud platforms. Therefore, power providers shifting to smart grids need to restore customers' confidence by addressing security issues. These concerns can best be addressed before being integrated to cloud platform which support smart grids.

\section{Conclusions}

This study covers only some selected issues related to the performance of the wireless communication, and how to make it more efficient and better utilized. There is still much work to be done in the future to improve the efficiency of interference cancellation. In this analysis, smart clouds and energy efficiency have been explored. Smart grid combines tradition grid with communicational technologies and advanced sensors to realize energy efficiency. Consumers can access usage information through smart meters. It also allows the integration of renewable energy resources into the power systems thus meeting energy demand without energy and resource wastage. Smart grid allow effective real-time diagnosis and load balance through automated outage management, self-activating, and self healing which encourage energy efficiency. Cloud platform is necessary in supporting smart software architecture for smart grids. Clouds provide elastic and scalable resources necessary for the design of smart software infrastructure necessary for smart grids. Data and information can easily be stored in cloud platforms and shared among utilities, third party software 
applications, and consumers. Because of the increased energy demand and use of renewable resources, energy efficient smart grid devices have to be built on smart software architecture which is supported by clouds. Advanced meter infrastructure has emerged as major smart software necessary in ensuring energy efficiency. Issues of security and privacy have emerged as the major setback of using cloud platforms to support smart software architecture necessary for smart grids.

\section{References}

1. Balaraman, S. 2012. Key considerations for designing low cost, energy efficient smart grid devices. [Online]. Available at: http://www.rtcmagazine.com/articles/view/102619 (accessed 19 January 2013).

2. Birman, P K., Ganesh, L., \& van Renesse, R. 2011. Running smart grid control software on cloud computing architectures. [Online]. Available at: (accessed 19 January 2013). http://www.cs.cornell.edu/projects/quicksilver/public_pdfs/SmartGrid-final.pdf

3. Drake, J., Najewicz, D, Watts,W, \& General Electric Company. 2010. Energy efficiency comparisons of wireless communication technology options for smart grid enabled devices. [Online]. Available at: http://energypriorities.com/library/ge_zigbee_vs_wifi_101209.pdf (accessed 19 January 2013).

4. Michaels, H., \& Donnelly, K. 2010. Architecting the smart grid for energy efficiency. Massachusetts Institute of Technology.

5. Pérez, J, Díaz, J \& González, E. 2012. Designing and simulating smart grids. Technical University of Madrid (UPM), Spain

6. Potter, C W, Archambault, A \& Westrick, K. 2008. Building a smarter smart grid through better renewable energy information. [Online]. Available at: http://c0402442.cdn.cloudfiles.rackspacecloud.com/static/ttcms/1.0.0.44/us/docum ents/publications/IEEE_Smart_Grid_Final.pdf (accessed 19 January 2013).

7. Simmhan, Y, Giakkoupis, M, Cao, B \& Prasanna, V. 2011. On using cloud platforms in a software architecture for smart energy grids. University of Southern California, Los Angeles.

8. Simmhan, Y, Giakkoupis, M, Cao, B \& Prasanna, V. 2011a. An analysis of security and privacy issues in smart. University of Southern California, Los Angeles. 